\title{
ANSWER TO A PROBLEM OF MILLER
}

\section{CHEN YUEQING}

(Communicated by Irwin Kra)

\begin{abstract}
In this note we give an affirmative answer to a problem of $\mathrm{S}$. Miller. Our method is very simple.
\end{abstract}

Let $w(z)$ be a regular function in unit disc $|z|<1$ with $w(0)=0$. Thanks to Schwarz' Lemma, it is easy to deduce $|w(z)|<1$ if $\left|w(z)+z w^{\prime}(z)\right|<1(|z|<1)$. In 1978 , S. S. Miller and P. T. Mocanu [1] showed that it is true that $\mid w(z)+z w^{\prime}(z)+$ $z^{2} w^{\prime \prime}(z) \mid<1$ implies $|w(z)|<1$.

Naturally one would ask if it is true that $\left|w(z)+z w^{\prime}(z)+\cdots+z^{n} w^{(n)}(z)\right|<1$ implies $|w(z)|<1(|z|<1)$ for all positive integers $n$. It was put forward by Miller [2] in 1979. In this note we give an affirmative answer to it. ${ }^{1}$

THEOREM. Let $w(z)$ be a regular function in unit disc $|z|<1$ with $w(0)=0$ and satisfy the following condition

$$
\left|w(z)+z w^{\prime}(z)+\cdots+z^{n} w^{(n)}(z)\right|<1 \quad(\text { for some integer } n \geq 2)
$$

then

$$
|w(z)|<\sqrt{\sum_{k=1}^{\infty} \frac{1}{p_{k}^{2}}}<0.554
$$

where

$$
p_{k}=\left\{\begin{array}{l}
1+k+k(k-1)+\cdots+k !, \quad \text { when } k \leq n, \\
1+k+k(k-1)+\cdots+k(k-1) \cdots(k-n+1), \quad \text { when } k>n .
\end{array}\right.
$$

REMARK. As noted by Goldstein et al. [3], the best-possible bound in (2) is $\frac{1}{2}$, which is sharp for $w(z)=\frac{1}{2} z$.

PrOOF. Let

$$
w(z)=\sum_{k=1}^{\infty} a_{k} z^{k}
$$

then

$$
w(z)+z w^{\prime}(z)+\cdots+z^{n} w^{(n)}(z)=\sum_{k=1}^{\infty} p_{k} a_{k} z^{k}
$$

Received by the editors June 23, 1987 and, in revised form, September 30, 1987.

1980 Mathematics Subject Classification. Primary 30A10.

${ }^{1}$ After finishing this note, which had been presented at the 4th Chinese National Complex Function Theory Meeting held in August, 1984, I learned that M. Goldstein, R. R. Hall, J. SheilSmall, and H. L. Smith, [3] have solved a considerably more general problem. Very recently, we came across a paper by Sakari Topilla [4], which also gives an affirmative answer to this problem, but I still think this note is interesting because of its simplicity. 
where $p_{k}$ is defined by (3). From (1) and Littlewood's subordination theorem we have

$$
\sum_{k=1}^{\infty} p_{k}^{2}\left|a_{k}\right|^{2} \leq 1
$$

Making use of Cauchy's inequality we derive

$$
|w(z)|<\sum_{k=1}^{\infty}\left|a_{k}\right| \leq \sqrt{\left(\sum_{k=1}^{\infty} \frac{1}{p_{k}^{2}}\right)\left(\sum_{k=1}^{\infty} p_{k}^{2}\left|a_{k}\right|^{2}\right)} \leq \sqrt{\sum_{k=1}^{\infty} \frac{1}{p_{k}^{2}}} .
$$

So the first part of (2) is obtained.

From the definition of $p_{k}$ it is easy to see

$$
p_{k} \geq 1+k+k(k-1)=1+k^{2} \text {. }
$$

Applying the integral estimate

$$
\sum_{k=n+1}^{\infty} \frac{1}{\left(1+k^{2}\right)^{2}}<\int_{n}^{\infty} \frac{d x}{\left(1+x^{2}\right)^{2}}=\frac{1}{2}\left(\frac{\pi}{2}-\operatorname{arctg} n\right)-\frac{n}{2\left(n^{2}+1\right)}
$$

to $\sum_{k=11}^{\infty} 1 / p_{k}^{2}$, we obtain

$$
\sum_{k=1}^{\infty} \frac{1}{p_{k}^{2}} \leq \sum_{k=1}^{10} \frac{1}{\left(1+k^{2}\right)^{2}}+\sum_{k=11}^{\infty} \frac{1}{\left(1+k^{2}\right)^{2}}<0.3068827
$$

This inequality, combining with (4), gives

$$
|w(z)|<\sqrt{\sum_{k=1}^{\infty} \frac{1}{p_{k}^{2}}}<0.5539699
$$

The theorem is proved.

\section{BIBLIOGRAPHY}

1. S. S. Miller and P. T. Mocanu, Second order differential inequalities in the complex plane, J. Math. Anal. Appl. 65 (1978), 289-305.

2. D. A. Brannen and J. G. Clunie, Aspects of contemporary complex analysis, Academic Press, London, 1980 , p. 554.

3. M. Goldstein, R. R. Hall, T. Sheil-Small, and H. Smith, Convexity preservation of inverse Euler operators and a problem of S. Miller, Bull. London Math. Soc. 14 (1982), 537-541.

4. Sakari Toppila, Solutions of problems of Miller and Rubel, Ann. Acad. Sci. Fenn AI Math. 8 (1983), 369-370.

Department of Mathematics, hua Chiao University, Qiangzhow, Fujian, 